\title{
Exigências térmicas e estimativa do número de gerações dos biótipos "milho" e "arroz" de Spodoptera frugiperda
}

\author{
Gustavo Rossato Busato(1), Anderson Dionei Grützmacher ${ }^{(1)}$, Mauro Silveira Garcia(1), \\ Fabrizio Pinheiro Giolo ${ }^{(1)}$, Moisés João Zotti ${ }^{(1)}$ e Juliana de Magalhães Bandeira ${ }^{(1)}$
}

(1)Universidade Federal de Pelotas, Fac. de Agronomia “Eliseu Maciel”, Dep. de Fitossanidade, Caixa Postal 354, CEP 96010-900 Pelotas, RS.
E-mail: grbusato@hotmail.com, adgrutzmacher@uol.com.br, msgarcia@ufpel.tche.br, fgiolo.faem@ufpel.tche.br, mzotti.faem@ufpel.tche.br

\begin{abstract}
Resumo - O objetivo deste trabalho foi avaliar o efeito da temperatura sobre a biologia dos biótipos "milho" e “arroz” de Spodoptera frugiperda (J.E. Smith, 1797) (Lepidoptera: Noctuidae) e estimar o número de gerações por ano em laboratório e campo. Foram coletadas lagartas de quatro populações de $S$. frugiperda no Estado do Rio Grande do Sul, identificadas eletroforeticamente como os biótipos “milho” e "arroz”, em áreas isoladas, distanciadas entre si em mais de $300 \mathrm{~km}$, produtoras de milho e arroz irrigado e em áreas adjacentes, que produzem milho e arroz irrigado lado a lado. A temperatura mais adequada para o desenvolvimento dos dois biótipos foi $25^{\circ} \mathrm{C}$. Em laboratório, podem ser obtidas 11,0 e 11,3 gerações ano-1 do biótipo “milho” proveniente de áreas isoladas e adjacentes, respectivamente. Foram estimadas 12,1 gerações ano-1 do biótipo "arroz" quando proveniente de áreas isoladas e 12,2 gerações ano ${ }^{-1}$ quando proveniente de áreas adjacentes. Em campo, estimou-se a ocorrência de 8,3 e 6,1 gerações ano ${ }^{-1}$ do biótipo "milho", respectivamente, em áreas isoladas e áreas adjacentes e 8,4 e 7,0 gerações ano-1 do biótipo "arroz”, respectivamente, em áreas isoladas e áreas adjacentes. Em áreas adjacentes, o biótipo “arroz” apresenta uma geração a mais ao longo de um ano em relação ao biótipo "milho”.
\end{abstract}

Termos para indexação: Insecta, lagarta-do-cartucho, lagarta-da-folha, biologia, biótipos.

\section{Thermal requirements and estimate of the number of generations of biotypes "corn" and "rice" of Spodoptera frugiperda}

\begin{abstract}
The objective of this work was to evaluate the effect of the temperature on the biology of the biotypes “corn" and "rice” of Spodoptera frugiperda (J.E. Smith, 1797) (Lepidoptera: Noctuidae) and to estimate the number of generations per year in laboratory and field. Caterpillars of four populations of $S$. frugiperda were collected in Rio Grande do Sul State, Brazil, identified by electrophoresis as the biotypes "corn" and "rice” in isolated areas (spaced for more than $300 \mathrm{~km}$ ), areas of corn and irrigated rice production, as well as in adjacent areas that produces corn and rice irrigated side by side. The most appropriate temperature for the development of the two biotypes was $25^{\circ} \mathrm{C}$. In laboratory, a number between 11.0 and 11.3 generations year ${ }^{-1}$ for the biotype "corn" can be obtained, originating from isolated and adjacent areas, respectively, and, for the biotype "rice", 12.1 and 12.2 generations year ${ }^{-1}$ when originating from isolated and adjacent areas, respectively. In the field, the occurrence of 8.3 and 6.1 generations year ${ }^{-1}$ for the biotype "corn" was estimated, respectively, in isolated and adjacent areas and 8.4 and 7.0 generations year-1 for the "rice" biotype, respectively, in isolated and adjacent areas. In adjacent areas, the "rice” biotype presents an extra generation per year in relation to the "corn” biotype.
\end{abstract}

Index terms: Insecta, fall armyworm, biology, biotypes.

\section{Introdução}

A cultura do milho e do arroz irrigado é fundamental para o desenvolvimento socioeconômico do Estado do Rio Grande do Sul. Porém, o ataque de pragas desfolhadoras tem causado significativa redução na produtividade. Entre os insetos fitófagos que atacam as culturas, Spodoptera frugiperda (J.E. Smith, 1797)
(Lepidoptera: Noctuidae) é considerada uma das pragas mais prejudiciais (Martins \& Botton, 1996; Grützmacher et al., 2000).

No milho, é conhecida como lagarta-do-cartucho. As lagartas jovens consomem parte das folhas e mantêm a epiderme intacta, aparentando o sintoma de "raspagem", nesta cultura. As lagartas maiores perfuram as folhas e se desenvolvem no cartucho do milho, podendo também 
broquear a base da planta e atacar a espiga, à semelhança de outras lagartas (Grützmacher et al., 2000). As plantas infestadas sofrem os maiores danos na fase de 8 a 10 folhas, com redução de $18,7 \%$ no rendimento (Cruz \& Turpin, 1982).

No arroz irrigado, é conhecida como lagarta-da-folha e alimenta-se de plantas novas, antes da inundação definitiva dos arrozais, consumindo-as completamente. Em determinados anos, atinge níveis populacionais elevados, podendo destruir totalmente a lavoura. As lagartas alimentam-se, preferencialmente, de plantas de capimarroz (Echinochloa spp.), passando a atacar o arroz após a eliminação das invasoras por herbicidas (Martins \& Botton, 1996).

Inicialmente, $S$. frugiperda foi considerada uma espécie polífaga, com 23 famílias de plantas hospedeiras (Luginbill, 1928). Entretanto, Pashley (1986) sugeriu a sua divisão em dois biótipos ("milho" e "arroz"), com base na diferenciação genética. O estudo da compatibilidade reprodutiva indicou a existência de isolamento unidirecional (Pashley \& Martin, 1987). Atualmente, de acordo com Drès \& Mallet (2002), tais biótipos representam espécies crípticas associadas às plantas hospedeiras.

No Brasil, e em especial no Estado do Rio Grande do Sul, estudos iniciais evidenciaram a possibilidade de existirem os biótipos "milho" e "arroz" de $S$. frugiperda (Busato et al., 2002). Recentemente, Busato et al. (2003) confirmaram a hipótese, tendo sido detectadas diferenças fenotípicas e genotípicas entre biótipos associados às plantas hospedeiras.

A constatação da existência de biótipos de S. frugiperda é fundamental na implementação do manejo do inseto, pois pode haver um comportamento diferenciado na suscetibilidade a inseticidas (Pashley et al., 1987a) e na resistência de plantas aos biótipos (Pashley et al., 1987b), bem como na seleção da planta hospedeira para oviposição (Whitford et al., 1988).

Vários fatores podem ser determinantes e devem ser incluídos num modelo de previsão de ocorrência de um inseto. Dentre os componentes de modelos matemáticos de previsão de ocorrência de pragas, a temperatura é, certamente, um dos que mais se destaca. Em função das necessidades térmicas dos insetos, a temperatura influencia a possibilidade de ocorrência de maiores ou menores populações num determinado local (Haddad et al., 1999).
Até o momento, não foi investigada a influência da temperatura no ciclo evolutivo dos biótipos de $S$. frugiperda, com o intuito de determinar o número de gerações anuais e os processos de sobrevivência sob condições climáticas adversas. O conhecimento das necessidades térmicas dos insetos-praga permite a formulação de estratégias para interromper o desenvolvimento das futuras gerações no campo, mediante medidas de controle planejadas. Além disso, possibilita a organização da produção e armazenamento de insetos em laboratório, permitindo a realização de estudos básicos durante todo o ano.

Este trabalho teve como objetivo avaliar o efeito da temperatura sobre a biologia dos biótipos "milho" e "arroz" de $S$. frugiperda e estimar o número de gerações por ano em laboratório e no campo.

\section{Material e Métodos}

O experimento foi desenvolvido no Dep. de Fitossanidade, Fac. de Agronomia “Eliseu Maciel”, Universidade Federal de Pelotas, em Capão do Leão, RS.

Lagartas de quarto e quinto ínstar de quatro populações de $S$. frugiperda, identificadas eletroforeticamente como biótipos “milho" e "arroz” (Busato et al., 2003), foram coletadas em áreas isoladas, distanciadas entre si em mais de 300 km, nos municípios de Santa Rosa e de Uruguaiana, RS, produtores tradicionais de milho e arroz irrigado, respectivamente, e em áreas adjacentes no Município de Pelotas, RS, que produz milho e arroz irrigado lado a lado. As lagartas coletadas foram criadas sobre folhas do respectivo hospedeiro até a pupação e nas gerações subseqüentes foram mantidas em dieta artificial de Greene et al. (1976) modificada. O método de criação foi o descrito por Parra (2001), utilizando-se tubos de vidro de fundo chato $(2,5 \mathrm{~cm}$ de diâmetro $\mathrm{x}$ $8,5 \mathrm{~cm}$ de altura).

As diferentes fases de desenvolvimento dos biótipos de $S$. frugiperda foram estudadas nas temperaturas de 18, 22, 25, 28, 30 e $32^{\circ} \mathrm{C}$, em câmaras climatizadas com umidade relativa de $70 \pm 15 \%$ e fotófase de 14 horas.

Na determinação da duração e viabilidade da fase de ovo, as posturas em massa provenientes da criação da terceira geração de laboratório foram separadas, utilizando-se o método de Gross et al. (1981). Em cada temperatura, foram incubados 30 ovos em tubos de vidro de fundo chato, contendo um pedaço de papel-filtro umedecido com água destilada, num total de 20 repetições. O desenvolvimento embrionário foi acompanhado até o momento da eclosão. 
No estudo das fases de lagarta, pré-pupa e pupa, em cada uma das temperaturas, foram individualizadas 150 lagartas recém-eclodidas (da criação estoque) de cada biótipo de $S$. frugiperda, em tubos de vidro de fundo chato contendo dieta artificial. Diariamente, observou-se o desenvolvimento e a mortalidade, de modo a se obter a duração e viabilidade das fases e do período ovo-adulto.

O delineamento experimental foi inteiramente casualizado. Nas análises estatísticas foi utilizado o programa "Genes" (Cruz, 2001) e as médias foram comparadas pelo teste de Tukey a 5\% de probabilidade.

Os métodos para a estimativa do limiar térmico inferior ( $\mathrm{Tb}$ ) podem ser encontrados em Haddad et al. (1999). Entre eles, o método da hipérbole é o mais comumente utilizado na estimativa da Tb e da constante térmica (K) de insetos (Campbell et al., 1974).

Por meio da temperatura considerada ideal, estimouse o número de gerações anuais em laboratório, pela fórmula da constante térmica. Com base nas normais térmicas das três regiões do RS, foi estimado o número provável de gerações anuais dos biótipos de $S$. frugiperda a campo. De acordo com Silveira Neto et al. (1976) e Cividanes (2000), um dos métodos mais utilizados na estimativa do número de graus-dia (GD) baseia-se nas temperaturas máxima $\left(T_{\text {máx }}\right)$ e mínima $\left(\mathrm{T}_{\text {mín}}\right)$ registradas e no limite térmico inferior $(\mathrm{Tb})$.

\section{Resultados e Discussão}

O efeito da temperatura sobre a duração do período embrionário foi significativo (Tabela 1). A duração da fase de ovo decresceu consideravelmente com o au-

Tabela 1. Duração e viabilidade da fase de ovo ( \pm EP) dos biótipos “milho" e "arroz" de Spodoptera frugiperda provenientes de áreas isoladas e adjacentes do Rio Grande do Sul, em diferentes temperaturas ${ }^{(1)}$.

\begin{tabular}{|c|c|c|c|c|}
\hline \multirow{3}{*}{$\begin{array}{c}\text { Temperatura } \\
\left({ }^{\circ} \mathrm{C}\right)\end{array}$} & \multicolumn{4}{|c|}{ Local de coleta dos biótipos de $S$. frugiperda } \\
\hline & \multicolumn{2}{|c|}{ Áreas isoladas } & \multicolumn{2}{|c|}{ Áreas adjacentes } \\
\hline & "Milho" & "Arroz" & "Milho" & "Arroz" \\
\hline & \multicolumn{4}{|c|}{ Duração (dias) } \\
\hline 18 & $4,8 \pm 0,141 \mathrm{a}$ & $4,7 \pm 0,171 \mathrm{a}$ & $4,7 \pm 0,135 \mathrm{a}$ & $4,7 \pm 0,204 \mathrm{a}$ \\
\hline 22 & $4,0 \pm 0,038 \mathrm{~b}$ & $3,7 \pm 0,058 b$ & $3,9 \pm 0,036 b$ & $3,7 \pm 0,021 \mathrm{~b}$ \\
\hline 25 & $2,6 \pm 0,106 \mathrm{c}$ & $2,4 \pm 0,055 \mathrm{c}$ & $2,5 \pm 0,068 \mathrm{c}$ & $2,4 \pm 0,063 c$ \\
\hline 28 & $2,3 \pm 0,058 \mathrm{~cd}$ & $2,2 \pm 0,046 \mathrm{~d}$ & $2,3 \pm 0,068 \mathrm{~cd}$ & $2,2 \pm 0,043 \mathrm{~d}$ \\
\hline 30 & $2,1 \pm 0,039 \mathrm{~d}$ & $2,1 \pm 0,072 \mathrm{~d}$ & $2,1 \pm 0,036 \mathrm{~d}$ & $2,1 \pm 0,042 d$ \\
\hline 32 & $2,0 \pm 0,007 \mathrm{~d}$ & $2,0 \pm 0,007 \mathrm{~d}$ & $2,0 \pm 0,006 \mathrm{c}$ & $2,0 \pm 0,007 \mathrm{~d}$ \\
\hline \multicolumn{5}{|c|}{ Viabilidade $(\%)$} \\
\hline 18 & $72,7 \pm 3,660 \mathrm{c}$ & $72,3 \pm 3,770 \mathrm{c}$ & $67,7 \pm 3,299 \mathrm{c}$ & $70,8 \pm 4,203 \mathrm{c}$ \\
\hline 22 & $90,3 \pm 3,131 \mathrm{ab}$ & $88,0 \pm 3,544 \mathrm{ab}$ & $88,5 \pm 3,081 \mathrm{ab}$ & $91,3 \pm 3,118 \mathrm{ab}$ \\
\hline 25 & $95,7 \pm 1,635 \mathrm{a}$ & $98,3 \pm 0,601 \mathrm{a}$ & $96,8 \pm 1,166 \mathrm{a}$ & $98,2 \pm 0,644 \mathrm{a}$ \\
\hline 28 & $89,8 \pm 4,673 a b$ & $90,7 \pm 3,091 \mathrm{ab}$ & $90,9 \pm 4,663 \mathrm{ab}$ & $91,9 \pm 3,126 \mathrm{ab}$ \\
\hline 30 & $84,7 \pm 4,301 \mathrm{abc}$ & $87,6 \pm 3,942 \mathrm{ab}$ & $79,7 \pm 3,792 \mathrm{c}$ & $85,4 \pm 4,204 \mathrm{abc}$ \\
\hline 32 & $78,6 \pm 5,034 \mathrm{bc}$ & $76,5 \pm 5,218 \mathrm{bc}$ & $73,1 \pm 4,979 \mathrm{c}$ & $79,1 \pm 5,117 \mathrm{bc}$ \\
\hline
\end{tabular}

(1)Médias seguidas pela mesma letra nas colunas não diferem entre si pelo teste de Tukey a 5\% de probabilidade. mento da temperatura, variando de 4,8 dias $\left(18^{\circ} \mathrm{C}\right)$ a 2,0 dias $\left(32^{\circ} \mathrm{C}\right)$. A $25^{\circ} \mathrm{C}$, variou de 2,4 a 2,6 dias. Resultados similares foram obtidos por Ferraz (1982), ao estudar a biologia de $S$. frugiperda em dieta à base de feijão e levedura de cerveja, nas temperaturas de 20, 25,30 e $35^{\circ} \mathrm{C}$, variando entre 5,1 dias $\left(20^{\circ} \mathrm{C}\right)$ e 2,0 dias $\left(35^{\circ} \mathrm{C}\right)$. Os valores diferem dos obtidos por Nalim (1991), ao estudar o desenvolvimento de $S$. frugiperda em dieta à base de feijão, levedura de cerveja e germe de trigo a $25^{\circ} \mathrm{C}$ (2,9 dias) e por Souza (1999), ao estudar a biologia de $S$. frugiperda em dieta à base de feijão, levedura de cerveja e germe de trigo em três tipos de recipientes de criação a $25^{\circ} \mathrm{C}$ (2,0 dias).

A viabilidade mínima da fase de ovo foi de $67,7 \%$ e a máxima, de $98,3 \%$, sendo superior a $25^{\circ} \mathrm{C}$, porém, não diferindo estatisticamente, quando provenientes de áreas isoladas, dos valores de $22^{\circ} \mathrm{C}, 28^{\circ} \mathrm{C}$ e $30^{\circ} \mathrm{C}$ em ambos biótipos de $S$. frugiperda e, quando provenientes de áreas adjacentes dos valores de $25^{\circ} \mathrm{C}$ e $28^{\circ} \mathrm{C}$ no biótipo "milho" e de $22^{\circ} \mathrm{C}, 28^{\circ} \mathrm{C}$ e $30^{\circ} \mathrm{C}$ no biótipo "arroz" (Tabela 1). Os resultados encontram-se próximos dos valores relatados por Ferraz (1982), que constatou uma variação de $52,7 \%\left(35^{\circ} \mathrm{C}\right)$ a $92,0 \%\left(20^{\circ} \mathrm{C}\right)$. Porém, os valores diferem dos obtidos por Nalim (1991) e Souza (1999) a $25^{\circ} \mathrm{C}$, respectivamente, $86,9 \%$ e $81,2 \%$. Provavelmente, esta diferença esteja associada à menor perda de água que ocorre no tubo de vidro de fundo chato em relação à placa de Petri, utilizada pelos autores.

A Tb da fase de ovo do biótipo "milho" foi de $9,3^{\circ} \mathrm{C} \mathrm{e}$ $8,7^{\circ} \mathrm{C}$, respectivamente, quando provenientes de áreas isoladas e adjacentes e de $8,1^{\circ} \mathrm{C}$, do biótipo "arroz", independentemente do local de coleta (Tabela 2). Estes resultados encontram-se próximos do relatado por Ferraz (1982), que foi de $10,0^{\circ} \mathrm{C}$.

Na fase de larva, foi observada diferença significativa entre as temperaturas avaliadas, havendo redução da duração com o aumento térmico (Tabela 3). O período larval teve duração máxima de 41,9 dias a $18^{\circ} \mathrm{C}$ e mínima de 11,1 dias a $32^{\circ} \mathrm{C}$, e a $25^{\circ} \mathrm{C}$ variou de 13,2 a 16,5 dias. Os resultados obtidos encontram-se próximos dos relatados por Ferraz (1982), variando entre 33,4 dias a $20^{\circ} \mathrm{C}$ e 12,3 dias a $35^{\circ} \mathrm{C}$. Nalim (1991) constatou a $25^{\circ} \mathrm{C}$ que a duração da fase foi de 17,3 dias. De acordo com Parra (1991), a quantidade e qualidade do alimento consumido na fase larval afeta, entre outros aspectos, o seu tempo de desenvolvimento. Assim, a variação da duração verificada entre os autores pode ser atribuída à fonte alimentar utilizada na criação do inseto. 
A viabilidade mínima da fase de larva do biótipo "arroz" foi de 55,3\% e a máxima, de 96,7\%, sendo significativamente superior a $25^{\circ} \mathrm{C}$, porém, não diferindo quando provenientes de áreas isoladas dos valores de 18, 22, 28 e $30^{\circ} \mathrm{C}$ e, quando provenientes de áreas adjacentes dos valores de $28^{\circ} \mathrm{C}$ (Tabela 3). Os valores obtidos encontram-se acima dos valores relatados por Ferraz (1982), que constatou uma variação de $55,0 \%\left(35^{\circ} \mathrm{C}\right)$ a $72,5 \%\left(20^{\circ} \mathrm{C}\right.$ e $\left.25^{\circ} \mathrm{C}\right)$. Entretanto, os valores obtidos encontram-se na faixa dos relatados por Nalim (1991) a $25^{\circ} \mathrm{C}(98,3 \%)$. De modo geral, as temperaturas extre-

Tabela 2. Temperatura base (Tb), constante térmica (K) e coeficiente de determinação $\left(\mathrm{R}^{2}\right)$ das diferentes fases de desenvolvimento e estimativa do número de gerações anuais no laboratório a $25^{\circ} \mathrm{C}$ (NGL) e no campo (NGC) dos biótipos "milho" e "arroz" de Spodoptera frugiperda provenientes de áreas isoladas e adjacentes do Rio Grande do Sul.

\begin{tabular}{|c|c|c|c|c|c|}
\hline \multirow{3}{*}{$\begin{array}{l}\text { Fase de } \\
\text { desenvolvimento }\end{array}$} & \multirow[t]{3}{*}{ Variável } & \multicolumn{4}{|c|}{ Local de coleta dos biótipos de $S$. frugiperda } \\
\hline & & \multicolumn{2}{|c|}{ Áreas isoladas } & \multicolumn{2}{|c|}{ Áreas adjacentes } \\
\hline & & "Milho" & "Arroz" & "Milho" & "Arroz" \\
\hline \multirow[t]{3}{*}{ Ovo } & $\mathrm{Tb}\left({ }^{\circ} \mathrm{C}\right)$ & 9,3 & 8,1 & 8,7 & 8,1 \\
\hline & $\mathrm{K}$ (GD) & 44,2 & 45,6 & 45,2 & 45,6 \\
\hline & $\mathrm{R}^{2}(\%)$ & 96,2 & 93,9 & 95,2 & 93,9 \\
\hline \multirow[t]{3}{*}{ Larva } & $\mathrm{Tb}\left({ }^{\circ} \mathrm{C}\right)$ & 8,2 & 8,1 & 9,0 & 8,1 \\
\hline & $\mathrm{K}$ (GD) & 297,2 & 270,8 & 266,3 & 249,6 \\
\hline & $\mathrm{R}^{2}(\%)$ & 92,0 & 96,7 & 96,5 & 96,5 \\
\hline \multirow[t]{3}{*}{ Pré-Pupa } & $\mathrm{Tb}\left({ }^{\circ} \mathrm{C}\right)$ & 13,9 & 11,7 & 14,4 & 12,6 \\
\hline & $\mathrm{K}(\mathrm{GD})$ & 19,7 & 26,2 & 22,8 & 26,4 \\
\hline & $\mathrm{R}^{2}(\%)$ & 95,4 & 95,0 & 96,6 & 96,2 \\
\hline \multirow[t]{3}{*}{ Pupa } & $\mathrm{Tb}\left({ }^{\circ} \mathrm{C}\right)$ & 13,4 & 10,9 & 11,9 & 10,8 \\
\hline & $\mathrm{K}(\mathrm{GD})$ & 122,9 & 140,4 & 136,2 & 132,6 \\
\hline & $\mathrm{R}^{2}(\%)$ & 96,5 & 95,5 & 94,4 & 98,1 \\
\hline \multirow[t]{3}{*}{ Ovo-adulto } & $\mathrm{Tb}\left({ }^{\circ} \mathrm{C}\right)$ & 10,9 & 9,6 & 10,6 & 9,5 \\
\hline & $\mathrm{K}$ (GD) & 463,0 & 470,5 & 464,6 & 444,9 \\
\hline & $\mathrm{R}^{2}(\%)$ & 97,1 & 93,6 & 97,5 & 95,5 \\
\hline NGL & & 11,0 & 12,1 & 11,3 & 12,2 \\
\hline $\mathrm{NGC}$ & & 8,3 & 8,4 & 6,1 & 7,0 \\
\hline
\end{tabular}

Tabela 3. Duração e viabilidade da fase de larva ( \pm EP) dos biótipos “milho" e "arroz” de Spodoptera frugiperda provenientes de áreas isoladas e adjacentes do Rio Grande do Sul, em diferentes temperaturas ${ }^{(1)}$.

\begin{tabular}{|c|c|c|c|c|}
\hline \multirow{3}{*}{$\begin{array}{c}\text { Temperatura } \\
\left({ }^{\circ} \mathrm{C}\right)\end{array}$} & \multicolumn{4}{|c|}{ Local de coleta dos biótipos de $S$. frugiperda } \\
\hline & \multicolumn{2}{|c|}{ Áreas isoladas } & \multicolumn{2}{|c|}{ Áreas adjacentes } \\
\hline & "Milho" & "Arroz" & "Milho" & "Arroz" \\
\hline & \multicolumn{4}{|c|}{ Duração (dias) } \\
\hline 18 & $38,4 \pm 0,284 a$ & $37,6 \pm 0,408 \mathrm{a}$ & $41,9 \pm 0,472 \mathrm{a}$ & $33,3 \pm 0,317 \mathrm{a}$ \\
\hline 22 & $18,8 \pm 0,217 \mathrm{~b}$ & $17,3 \pm 0,156 \mathrm{~b}$ & $18,4 \pm 0,352 b$ & $15,9 \pm 0,164 \mathrm{~b}$ \\
\hline 25 & $16,5 \pm 0,407 \mathrm{c}$ & $13,9 \pm 0,380 \mathrm{c}$ & $14,1 \pm 0,081 \mathrm{c}$ & $13,2 \pm 0,067 \mathrm{c}$ \\
\hline 28 & $14,5 \pm 0,141 \mathrm{~d}$ & $13,2 \pm 0,091 \mathrm{c}$ & $13,6 \pm 0,085 \mathrm{c}$ & $12,1 \pm 0,107 \mathrm{~cd}$ \\
\hline 30 & $14,3 \pm 0,134 \mathrm{~d}$ & $12,5 \pm 0,124 \mathrm{c}$ & $13,2 \pm 0,143 \mathrm{c}$ & $11,8 \pm 0,104 \mathrm{~d}$ \\
\hline 32 & $12,9 \pm 0,208 \mathrm{e}$ & $12,4 \pm 0,202 \mathrm{c}$ & $12,5 \pm 0,217 \mathrm{c}$ & $11,1 \pm 0,117 \mathrm{e}$ \\
\hline & \multicolumn{4}{|c|}{ Viabilidade (\%) } \\
\hline 18 & $80,7 \pm 3,235 b$ & $81,3 \pm 3,192 \mathrm{ab}$ & $62,7 \pm 3,963 \mathrm{c}$ & $62,0 \pm 3,976 \mathrm{~d}$ \\
\hline 22 & $78,7 \pm 3,356 b$ & $80,0 \pm 3,277 \mathrm{ab}$ & $69,3 \pm 3,778 b c$ & $74,7 \pm 3,563 \mathrm{~cd}$ \\
\hline 25 & $94,0 \pm 0,149 \mathrm{a}$ & $90,7 \pm 2,383 \mathrm{a}$ & $96,0 \pm 1,605 a$ & $96,7 \pm 1,471 \mathrm{a}$ \\
\hline 28 & $76,7 \pm 3,465 b$ & $80,7 \pm 3,235 \mathrm{ab}$ & $80,7 \pm 3,235 b$ & $88,7 \pm 2,597 \mathrm{ab}$ \\
\hline 30 & $68,0 \pm 3,822 b$ & $79,3 \pm 3,317 \mathrm{ab}$ & $80,0 \pm 3,277 \mathrm{~b}$ & $80,7 \pm 3,235 b c$ \\
\hline 32 & $55,3 \pm 5,475 \mathrm{c}$ & $68,7 \pm 3,800 \mathrm{~b}$ & $69,3 \pm 5,078 \mathrm{bc}$ & $77,3 \pm 2,153 \mathrm{bc}$ \\
\hline
\end{tabular}

${ }^{(1)}$ Médias seguidas pela mesma letra nas colunas não diferem entre si pelo teste de Tukey a $5 \%$ de probabilidade. mas foram mais prejudiciais ao desenvolvimento das lagartas, o que está de acordo com o observado em Spodoptera eridania (Cramer) (Foerster \& Dionísio, 1989), Pseudaletia sequax Franclemont (Foerster, 1996) e em Spodoptera cosmioides (Walker) (Bavaresco et al., 2002).

O limiar térmico inferior de desenvolvimento (Tb) da fase de lagarta do biótipo "milho" foi de $8,2^{\circ} \mathrm{C}$ e $9,0^{\circ} \mathrm{C}$, respectivamente, quando proveniente de áreas isoladas e adjacentes (Tabela 2). No biótipo “arroz”, foi de $8,1^{\circ} \mathrm{C}$, independentemente do local de coleta. O resultado obtido encontra-se próximo do relatado por Ferraz (1982), de $10,7^{\circ} \mathrm{C}$.

Na fase de pré-pupa, foi observada diferença significativa entre a maioria das temperaturas avaliadas, principalmente entre $18^{\circ} \mathrm{C}, 22^{\circ} \mathrm{C}$ e $25^{\circ} \mathrm{C}$, dependendo do biótipo, havendo redução da duração do período com o aumento da temperatura (Tabela 4). O período pré-pupal teve duração máxima de 4,8 dias a $18^{\circ} \mathrm{C}$ e a mínima, de 1,1 dia a $32^{\circ} \mathrm{C}$, e a $25^{\circ} \mathrm{C}$ variou de 1,8 a 2,4 dias. Os resultados obtidos encontram-se próximos dos valores relatados por Ferraz (1982), que constatou uma variação de 3,7 e 1,6 dias, respectivamente, a $20^{\circ} \mathrm{C}$ e $35^{\circ} \mathrm{C}$. Também encontram-se próximos dos obtidos por Nalim (1991) a $25^{\circ} \mathrm{C}$ (2,0 dias).

A viabilidade mínima da fase de pré-pupa foi de $63,8 \%$ e a máxima, de 99,2\%, sendo significativamente superior na faixa de $18^{\circ} \mathrm{C}$ a $30^{\circ} \mathrm{C}$, e semelhantes entre si (Tabela 4). Ferraz (1982) também constatou, nessa fase, elevada viabilidade - nas quatro temperaturas avalia-

Tabela 4. Duração e viabilidade da fase de pré-pupa ( $\pm \mathrm{EP})$ dos biótipos "milho" e "arroz" de Spodoptera frugiperda provenientes de áreas isoladas e adjacentes do Rio Grande do Sul, em diferentes temperaturas ${ }^{(1)}$.

\begin{tabular}{|c|c|c|c|c|}
\hline \multirow{3}{*}{$\begin{array}{c}\text { Temperatura } \\
\left({ }^{\circ} \mathrm{C}\right)\end{array}$} & \multicolumn{4}{|c|}{ Local de coleta dos biótipos de $S$. frugiperda } \\
\hline & \multicolumn{2}{|c|}{ Áreas isoladas } & \multicolumn{2}{|c|}{ Áreas adjacentes } \\
\hline & "Milho" & "Arroz" & "Milho" & "Arroz" \\
\hline & \multicolumn{4}{|c|}{ Duração (dias) } \\
\hline 18 & $3,6 \pm 0,157 \mathrm{a}$ & $4,3 \pm 0,167 \mathrm{a}$ & $4,8 \pm 0,155 \mathrm{a}$ & $4,6 \pm 0,278 a$ \\
\hline 22 & $3,0 \pm 0,153 b$ & $2,9 \pm 0,165 b$ & $3,2 \pm 0,185 b$ & $3,4 \pm 0,198 b$ \\
\hline 25 & $2,0 \pm 0,000 \mathrm{c}$ & $1,8 \pm 0,092 \mathrm{c}$ & $2,4 \pm 0,745 b c$ & $1,9 \pm 0,038 \mathrm{c}$ \\
\hline 28 & $1,3 \pm 0,071 \mathrm{~cd}$ & $1,5 \pm 0,081 \mathrm{c}$ & $1,7 \pm 0,105 \mathrm{~cd}$ & $1,7 \pm 0,127 \mathrm{c}$ \\
\hline 30 & $1,2 \pm 0,134 \mathrm{~cd}$ & $1,4 \pm 0,056 \mathrm{c}$ & $1,5 \pm 0,056 \mathrm{~cd}$ & $1,5 \pm 0,058 \mathrm{c}$ \\
\hline 32 & $1,1 \pm 0,051 \mathrm{~d}$ & $1,4 \pm 0,072 \mathrm{c}$ & $1,2 \pm 0,061 \mathrm{~d}$ & $1,4 \pm 0,045 \mathrm{c}$ \\
\hline & \multicolumn{4}{|c|}{ Viabilidade $(\%)$} \\
\hline 18 & $95,0 \pm 2,033 a$ & $92,6 \pm 2,376 a$ & $98,9 \pm 0,962 \mathrm{a}$ & $96,8 \pm 1,842 \mathrm{a}$ \\
\hline 22 & $93,6 \pm 2,359 a$ & $87,8 \pm 2,789 a$ & $94,2 \pm 2,297 \mathrm{a}$ & $92,9 \pm 2,444 a$ \\
\hline 25 & $95,0 \pm 0,018 \mathrm{a}$ & $96,2 \pm 1,643 a$ & $95,8 \pm 1,671 \mathrm{a}$ & $97,9 \pm 1,186 a$ \\
\hline 28 & $99,1 \pm 0,848 \mathrm{a}$ & $98,3 \pm 1,164 \mathrm{a}$ & $99,2 \pm 0,826 \mathrm{a}$ & $95,5 \pm 1,807 a$ \\
\hline 30 & $95,1 \pm 1,989 \mathrm{a}$ & $87,4 \pm 3,055 \mathrm{a}$ & $98,5 \pm 1,515 \mathrm{a}$ & $98,3 \pm 1,164 \mathrm{a}$ \\
\hline 32 & $73,5 \pm 5,162 \mathrm{~b}$ & $64,1 \pm 4,750 \mathrm{~b}$ & $75,0 \pm 5,058 \mathrm{~b}$ & $63,8 \pm 4,482 \mathrm{~b}$ \\
\hline
\end{tabular}

${ }^{(1)}$ Médias seguidas pela mesma letra nas colunas não diferem entre si pelo teste de Tukey a 5\% de probabilidade. 
das, foi superior a 90,7\%, fato também verificado por Nalim (1991) a $25^{\circ} \mathrm{C}(93,3 \%)$. Comportamento semelhante foi observado em S. cosmioides (Bavaresco et al., 2002), que apresentou maior redução da população na temperatura extrema superior.

O limiar térmico inferior de desenvolvimento (Tb) da fase de pré-pupa do biótipo "milho" foi de $13,9^{\circ} \mathrm{C}$ e $14,4^{\circ} \mathrm{C}$, respectivamente, quando provenientes de áreas isoladas e adjacentes. No biótipo “arroz”, foi de $11,7^{\circ} \mathrm{C}$ e $12,6^{\circ} \mathrm{C}$, respectivamente, quando provenientes de áreas isoladas e adjacentes (Tabela 2). Estes valores encontram-se acima do relatado por Ferraz (1982), que foi de $6,4^{\circ} \mathrm{C}$. A discrepância pode ser atribuída à curta duração da fase e à possibilidade do desenvolvimento ser semelhante em mais de uma temperatura.

O efeito da temperatura sobre a duração da fase pupal foi significativo (Tabela 5). A duração dessa fase variou de 30,4 dias $\left(18^{\circ} \mathrm{C}\right)$ a 6,6 dias $\left(32^{\circ} \mathrm{C}\right)$, e a $25^{\circ} \mathrm{C}$ variou de 8,8 a 12,6 dias. Os resultados obtidos encontram-se acima dos valores relatados por Ferraz (1982), que observou nos machos uma duração da fase pupal de 21,2 dias a $20^{\circ} \mathrm{C}$ e 5,9 dias a $35^{\circ} \mathrm{C}$. Valores similares aos obtidos neste trabalho são relatados por Nalim $(1999)$ a $25^{\circ} \mathrm{C}$ (11,0 dias). Porém, resultados discrepantes foram constatados por Souza (1999) a $25^{\circ} \mathrm{C}$ (17,1 dias).

A viabilidade mínima da fase de pupa foi de $71,6 \%$ e a máxima, de 98,5\% (Tabela 5). Quando provenientes de áreas isoladas foi significativamente superior a $25^{\circ} \mathrm{C}$, porém, no biótipo "milho", não diferiu dos valores a 18 , 22, 28 e $32^{\circ} \mathrm{C}$. Quando provenientes de áreas adjacen-

Tabela 5. Duração e viabilidade da fase de pupa ( \pm EP) dos biótipos “milho" e "arroz” de Spodoptera frugiperda provenientes de áreas isoladas e adjacentes do Rio Grande do Sul, em diferentes temperaturas ${ }^{(1)}$.

\begin{tabular}{|c|c|c|c|c|}
\hline \multirow{3}{*}{$\begin{array}{c}\text { Temperatura } \\
\left({ }^{\circ} \mathrm{C}\right)\end{array}$} & \multicolumn{4}{|c|}{ Local de coleta dos biótipos de $S$. frugiperda } \\
\hline & \multicolumn{2}{|c|}{ Áreas isoladas } & \multicolumn{2}{|c|}{ Áreas adjacentes } \\
\hline & "Milho" & "Arroz" & "Milho" & "Arroz" \\
\hline & \multicolumn{4}{|c|}{ Duração (dias) } \\
\hline 18 & $30,4 \pm 0,510 \mathrm{a}$ & $23,4 \pm 0,430 \mathrm{a}$ & $20,6 \pm 0,358 \mathrm{a}$ & $20,1 \pm 0,420 \mathrm{a}$ \\
\hline 22 & $12,9 \pm 0,233 b$ & $12,4 \pm 0,245 b$ & $12,7 \pm 0,254 \mathrm{~b}$ & $12,0 \pm 0,283 b$ \\
\hline 25 & $10,6 \pm 0,242 \mathrm{c}$ & $9,2 \pm 0,218 \mathrm{c}$ & $12,6 \pm 0,183 \mathrm{c}$ & $8,8 \pm 0,713 \mathrm{c}$ \\
\hline 28 & $7,9 \pm 0,121 \mathrm{~d}$ & $7,6 \pm 0,125 \mathrm{~cd}$ & $7,8 \pm 0,104 \mathrm{c}$ & $7,5 \pm 0,089 \mathrm{c}$ \\
\hline 30 & $7,2 \pm 0,166 \mathrm{~d}$ & $7,5 \pm 0,134 \mathrm{~cd}$ & $7,4 \pm 0,090 \mathrm{~cd}$ & $6,8 \pm 0,196 \mathrm{~d}$ \\
\hline \multirow[t]{2}{*}{32} & $7,1 \pm 0,256 \mathrm{~d}$ & $7,1 \pm 0,197 \mathrm{~d}$ & $6,8 \pm 0,150 \mathrm{~d}$ & $6,6 \pm 0,123 \mathrm{~d}$ \\
\hline & \multicolumn{4}{|c|}{ Viabilidade $(\%)$} \\
\hline 18 & $84,3 \pm 3,705 \mathrm{ab}$ & $81,4 \pm 3,675 b c$ & $82,8 \pm 3,853 b$ & $81,1 \pm 4,149 \mathrm{~b}$ \\
\hline 22 & $83,3 \pm 3,708 \mathrm{ab}$ & $81,7 \pm 4,090 \mathrm{bc}$ & $87,8 \pm 3,328 \mathrm{ab}$ & $89,4 \pm 3,030 \mathrm{ab}$ \\
\hline 25 & $92,5 \pm 0,022 \mathrm{a}$ & $94,5 \pm 2,033 \mathrm{a}$ & $94,2 \pm 1,997 \mathrm{a}$ & $96,5 \pm 1,552 \mathrm{a}$ \\
\hline 28 & $96,8 \pm 3,112 \mathrm{ab}$ & $88,2 \pm 2,966 \mathrm{~b}$ & $91,7 \pm 2,544 \mathrm{ab}$ & $94,1 \pm 1,733 \mathrm{a}$ \\
\hline 30 & $73,2 \pm 4,366 \mathrm{~b}$ & $80,8 \pm 3,883 \mathrm{bc}$ & $98,5 \pm 1,538 \mathrm{a}$ & $95,8 \pm 1,862 \mathrm{a}$ \\
\hline 32 & $86,9 \pm 4,128 \mathrm{ab}$ & $71,6 \pm 5,590 \mathrm{c}$ & $85,1 \pm 4,348 \mathrm{ab}$ & $91,9 \pm 3,195 \mathrm{ab}$ \\
\hline
\end{tabular}

${ }^{(1)}$ Médias seguidas pela mesma letra nas colunas não diferem entre si pelo teste de Tukey a 5\% de probabilidade. tes, foi significativamente superior a $25^{\circ} \mathrm{C}$ e $30^{\circ} \mathrm{C}$, porém, não diferindo dos valores a $22^{\circ} \mathrm{C}, 28^{\circ} \mathrm{C}$ e $32^{\circ} \mathrm{C}$ no biótipo “milho”, ao passo que no biótipo "arroz”, foi maior na faixa de $25^{\circ} \mathrm{C}$ a $30^{\circ} \mathrm{C}$, porém, não diferindo dos valores a $22^{\circ} \mathrm{C}$ e $32^{\circ} \mathrm{C}$. Ferraz (1982) constatou nas quatro temperaturas avaliadas viabilidade superior a $79,5 \%$. Nalim (1991) e Souza (1999) também constataram elevada viabilidade nessa fase (96,4\% e 90,3\%). Comportamento semelhante foi observado em $P$. sequax (Foerster, 1996) e em S. cosmioides (Bavaresco et al., 2002), havendo maior redução da população nas temperaturas extremas superior e inferior.

A Tb da fase de pupa do biótipo "milho" foi de $13,4^{\circ} \mathrm{C}$ e $11,9^{\circ} \mathrm{C}$, respectivamente, quando proveniente de áreas isoladas e adjacentes (Tabela 2). No biótipo “arroz”, foi de $10,9^{\circ} \mathrm{C}$ e $10,8^{\circ} \mathrm{C}$, respectivamente, quando proveniente de áreas isoladas e adjacentes. O resultado encontra-se próximo do relatado por Ferraz (1982), de $12,5^{\circ} \mathrm{C}$.

Quanto ao período ovo-adulto, foi observada diferença significativa entre as temperaturas avaliadas, havendo redução da duração do período com o aumento da temperatura (Tabela 6). Verificou-se variação de 77,3 dias a 21,0 dias, respectivamente, a $18^{\circ} \mathrm{C}$ e $32^{\circ} \mathrm{C}$, e a $25^{\circ} \mathrm{C}$, houve variação de 26,2 a 32,5 dias. Os resultados obtidos encontram-se próximos dos valores relatados por Nalim (1991) a $25^{\circ} \mathrm{C}$ (33,2 dias).

A viabilidade mínima do período ovo-adulto foi de 24,3\% e a máxima, de 89,7\% (Tabela 6). Quando provenientes de áreas isoladas, foi significativamente su-

Tabela 6. Duração e viabilidade do ciclo total (ovo-adulto) ( \pm EP) dos biótipos “milho” e "arroz” de Spodoptera frugiperda provenientes de áreas isoladas e adjacentes do Rio Grande do Sul, em diferentes temperaturas ${ }^{(1)}$.

\begin{tabular}{|c|c|c|c|c|}
\hline \multirow{3}{*}{$\begin{array}{c}\text { Temperatura } \\
\left({ }^{\circ} \mathrm{C}\right)\end{array}$} & \multicolumn{4}{|c|}{ Local de coleta dos biótipos de $S$. frugiperda } \\
\hline & \multicolumn{2}{|c|}{ Áreas isoladas } & \multicolumn{2}{|c|}{ Áreas adjacentes } \\
\hline & "Milho" & "Arroz" & "Milho" & "Arroz" \\
\hline & \multicolumn{4}{|c|}{ Duração (dias) } \\
\hline 18 & $77,3 \pm 0,621 \mathrm{a}$ & $69,5 \pm 0,523 \mathrm{a}$ & $71,9 \pm 0,496 \mathrm{a}$ & $61,3 \pm 0,561 \mathrm{a}$ \\
\hline 22 & $38,4 \pm 0,267 \mathrm{~b}$ & $35,9 \pm 0,267 \mathrm{~b}$ & $37,4 \pm 0,363 b$ & $34,7 \pm 0,313 b$ \\
\hline 25 & $32,5 \pm 0,272 \mathrm{c}$ & $27,7 \pm 0,181 \mathrm{c}$ & $31,9 \pm 0,222 \mathrm{c}$ & $26,2 \pm 0,128 \mathrm{c}$ \\
\hline 28 & $25,6 \pm 0,206 \mathrm{~d}$ & $24,3 \pm 0,155 \mathrm{~d}$ & $25,2 \pm 0,147 \mathrm{~d}$ & $22,9 \pm 0,117 \mathrm{~d}$ \\
\hline 30 & $24,7 \pm 0,163 \mathrm{de}$ & $23,2 \pm 0,206 \mathrm{~d}$ & $24,1 \pm 0,183 \mathrm{de}$ & $22,0 \pm 0,249 \mathrm{de}$ \\
\hline 32 & $22,9 \pm 0,279 \mathrm{e}$ & $22,7 \pm 0,322 \mathrm{~d}$ & $22,8 \pm 0,098 \mathrm{e}$ & $21,0 \pm 0,151 \mathrm{e}$ \\
\hline & \multicolumn{4}{|c|}{ Viabilidade $(\%)$} \\
\hline 18 & $47,2 \pm 1,683 b c$ & $49,4 \pm 1,576 b$ & $34,9 \pm 2,133 b c$ & $34,6 \pm 2,196 \mathrm{~d}$ \\
\hline 22 & $55,2 \pm 1,816 \mathrm{bc}$ & $44,2 \pm 1,716 \mathrm{~b}$ & $51,0 \pm 1,956 \mathrm{~b}$ & $56,4 \pm 1,808 \mathrm{c}$ \\
\hline 25 & $79,1 \pm 1,102 \mathrm{a}$ & $81,1 \pm 1,148 \mathrm{a}$ & $83,9 \pm 0,550 \mathrm{a}$ & $89,7 \pm 0,501 \mathrm{a}$ \\
\hline 28 & $59,4 \pm 1,683 b$ & $63,7 \pm 1,548 \mathrm{ab}$ & $66,7 \pm 1,477 \mathrm{a}$ & $73,2 \pm 1,198 \mathrm{ab}$ \\
\hline 30 & $40,2 \pm 2,135 \mathrm{~cd}$ & $49,3 \pm 1,889 b$ & $62,1 \pm 3,860 \mathrm{bc}$ & $64,6 \pm 1,415 b c$ \\
\hline 32 & $27,9 \pm 2,596 \mathrm{~d}$ & $24,3 \pm 2,486 \mathrm{c}$ & $32,3 \pm 2,137 \mathrm{c}$ & $35,8 \pm 2,153 \mathrm{~d}$ \\
\hline
\end{tabular}

(1)Médias seguidas pela mesma letra nas colunas não diferem entre si pelo teste de Tukey a 5\% de probabilidade. 
perior a $25^{\circ} \mathrm{C}$, porém, no biótipo “arroz”, não diferiu do valor a $28^{\circ} \mathrm{C}$. Quando provenientes de áreas adjacentes, foi significativamente superior a $25^{\circ} \mathrm{C}$ e $28^{\circ} \mathrm{C}$ no biótipo "milho", ao passo que no biótipo "arroz" foi maior a $25^{\circ} \mathrm{C}$, porém, não diferindo do valor a $28^{\circ} \mathrm{C}$. Os valores observados encontram-se próximos dos relatados por Nalim (1991) a $25^{\circ} \mathrm{C}(75,0 \%)$. Comportamento semelhante foi observado em $P$. sequax (Foerster, 1996) e em S. cosmioides (Bavaresco et al., 2002), havendo maior redução da população nas temperaturas extremas superior e inferior. Diante dos resultados, somente a $25^{\circ} \mathrm{C}$ a viabilidade foi superior ao mínimo de $75,0 \%$ preconizado por Singh (1983).

O limiar térmico inferior de desenvolvimento ( $\mathrm{Tb}$ ) do período ovo-adulto do biótipo "milho" foi de $10,9^{\circ} \mathrm{C}$ e $10,6^{\circ} \mathrm{C}$, respectivamente, quando proveniente de áreas isoladas e adjacentes (Tabela 2). No biótipo “arroz”, foi de $9,6^{\circ} \mathrm{C}$ e $9,5^{\circ} \mathrm{C}$, respectivamente, quando proveniente de áreas isoladas e adjacentes. Ferraz (1982), embora não tenha determinado a Tb para o período ovo-adulto, cita que a constante térmica (K) foi de 517,5 GD, evidenciando uma acentuada diferença, pois, no presente estudo obteve-se valores que variaram de 444,9 a 470,5 GD. De acordo com Silveira Neto et al. (1976), indiretamente, a temperatura afeta a alimentação dos insetos, de modo que a referida diferença pode ser atribuída especialmente à dieta utilizada.

Considerando que a duração do ciclo total foi relativamente baixa e que a viabilidade total está acima do preconizado, constata-se que a temperatura de $25^{\circ} \mathrm{C}$ é a mais adequada para o desenvolvimento dos biótipos "milho" e "arroz" de S. frugiperda, independentemente do local de coleta. Nesta temperatura, estimou-se 11,0 e 11,3 gerações por ano do biótipo "milho" proveniente de áreas isoladas e adjacentes, respectivamente, e, do biótipo “arroz", 12,1 gerações por ano quando proveniente de áreas isoladas e 12,2 gerações anuais, quando proveniente de áreas adjacentes.

Tomando-se por base os dados de constante térmica e normais climatológicas, estimou-se a ocorrência de 8,3 e 6,1 gerações por ano do biótipo “milho", respectivamente, em Santa Rosa (áreas isoladas) e Pelotas (áreas adjacentes) e 8,4 e 7,0 gerações por ano do biótipo “arroz", respectivamente, em Uruguaiana (áreas isoladas) e Pelotas (áreas adjacentes). Destaca-se o fato de o biótipo "arroz" apresentar, em áreas adjacentes, uma geração a mais ao longo de um ano em relação ao biótipo "milho".
A constatação da existência dos biótipos "milho" e "arroz" de S. frugiperda no Estado do Rio Grande do Sul e, em especial, no agroecossistema de várzea, evidencia um novo cenário para os entomologistas no âmbito da implementação do manejo integrado da praga nas culturas do milho e arroz irrigado. Nesses ambientes, deve-se sempre considerar a espécie da planta hospedeira, sendo este aspecto fundamental para a rotação de culturas. Isto porque, além das diferenças de performance nas plantas hospedeiras, em áreas onde coexistem (Município de Pelotas), estimou-se uma geração a mais do biótipo "arroz” ao longo de um ano.

\section{Conclusões}

1. A temperatura mais adequada para o desenvolvimento dos biótipos "milho" e "arroz" de $S$. frugiperda é $25^{\circ} \mathrm{C}$.

2. Em laboratório, podem ser obtidas 11,0 e 11,3 gerações por ano do biótipo "milho" proveniente de áreas isoladas e adjacentes, respectivamente, e do biótipo "arroz”, 12,1 gerações por ano, quando proveniente de áreas isoladas, e 12,2 gerações anuais, quando proveniente de áreas adjacentes.

3. No campo, podem ocorrer 8,3 e 6,1 gerações por ano do biótipo "milho", respectivamente, em áreas isoladas e áreas adjacentes, e 8,4 e 7,0 gerações por ano do biótipo “arroz”, respectivamente, em áreas isoladas e áreas adjacentes.

4. Em áreas adjacentes, o biótipo "arroz" apresenta uma geração a mais ao longo de um ano em relação ao biótipo "milho".

\section{Referências}

BAVARESCO, A.; GARCIA, M.S.; GRÜTZMACHER, A.D.; FORESTI, J.; RINGENBERG, R. Biologia e exigências térmicas de Spodoptera cosmioides (Walk.) (Lepidoptera: Noctuidae). Neotropical Entomology, v.31, p.49-54, 2002.

BUSATO, G.R.; GRÜTZMACHER, A.D.; GARCIA, M.S.; GIOLO, F.P.; MARTINS, A.F. Consumo e utilização de alimento por Spodoptera frugiperda (J.E. Smith) (Lepidoptera: Noctuidae) originária de diferentes regiões do Rio Grande do Sul, das culturas do milho e do arroz irrigado. Neotropical Entomology, v.31, p.525529, 2002.

BUSATO, G.R.; GRÜTZMACHER, A.D.; OLIVEIRA, A.C. de; ZIMMER, P.D.; KOPP, M.M.; VIEIRA, E.A.; MALONE, G. Caracterização genética de populações de Spodoptera frugiperda (J.E. Smith, 1797) (Lepidoptera: Noctuidae) provenientes das culturas do arroz irrigado e milho no Rio Grande do Sul através da técnica de AFLP. In: CONGRESSO BRASILEIRO DE ARROZ IRRIGADO, 3., REUNIÃO DA CULTURA DO ARROZ IRRIGADO, 25., 2003, Balneário Camburiú. Anais. Itajaí: EPAGRI, 2003. p.377-379. 
CAMPBELL, A.; FRAZER, B.D.; GILBERT, N.; GUTIERREZ, A.P.; MACKAUER, M. Temperature requirements of some aphids and their parasites. Journal of Applied Ecology, v.11, p.431-438, 1974.

CIVIDANES, F.J. Uso de graus-dias em entomologia: com particular referência ao controle de percevejos pragas da soja. Jaboticabal: Funep, 2000. 31p.

CRUZ, C.D. Programa Genes: aplicativo computacional em genética e estatística. Viçosa: Ed. Universidade Federal de Viçosa, 2001. 648p.

CRUZ, I.; TURPIN, F.T. Efeitos da Spodoptera frugiperda em diferentes estádios de crescimento da cultura do milho. Pesquisa Agropecuária Brasileira, v.17, p.355-359, 1982.

DRÈS, M.; MALLET, J. Host races in plant-feeding insects and their importance sympatric speciation. Philosophical Transactions: Biological Sciences, v.357, p.471-492, 2002.

FERRAZ, M.C.V.D. Determinação das exigências térmicas de Spodoptera frugiperda (J.E. Smith, 1797) (Lepidoptera: Noctuidae) em cultura de milho. 1982. 81p. Dissertação (Mestrado) - Escola Superior de Agricultura Luiz de Queiroz, Piracicaba, 1982.

FOERSTER, L.A. Efeito da temperatura no desenvolvimento das fases imaturas de Pseudaletia sequax Franclemont (Lepidoptera: Noctuidae). Anais da Sociedade Entomológica do Brasil, v.25, p.27-32, 1996.

FOERSTER, L.A.; DIONÍSIO, A.L.M. Necessidades térmicas de Spodoptera eridania (Cramer, 1782) (Lepidoptera: Noctuidae) em bracatinga (Mimosa scabrella Bentham (Leguminosae)). Anais da Sociedade Entomológica do Brasil, v.18, p.145-154, 1989.

GREENE, G.L.; LEPLA, N.C.; DICKERSON, W.A. Velvetbean caterpillar: a rearing procedure and artificial medium. Journal of Economic Entomology, v.69, p.488-497, 1976.

GROSS, H.R.; JOHNSON JUNIOR, R.; HARREL, E.A.; PERKINS, W.D. Method of separating fall armyworm eggs from masses. Journal of Economic Entomology, v.74, p.122-123, 1981.

GRÜTZMACHER, A.D.; MARTINS, J.F. da S.; CUNHA, U.S. da. Insetos-pragas das culturas do milho e sorgo no agroecossistema de várzea. In: PARFITT, J.M.B. Produção de milho e sorgo em várzea. Pelotas: Embrapa Clima Temperado, 2000. p.87-102.

HADDAD, M.L.; PARRA, J.R.P.; MORAES, R.C. Métodos para estimar os limites térmicos inferior e superior de desenvolvimento de insetos. Piracicaba: Fealq, 1999. 29p.

LUGINBILL, P. The fall armyworm. Technical Bulletin United States Department of Agriculture, v.34, p.1-91, 1928.
MARTINS, J.F. da S.; BOTTON, M. Controle de insetos da cultura do arroz. In: PESKE, S.T.; NEDEL, J.L.; BARROS, A.C.S.A. (Ed.). Produção de arroz irrigado. Pelotas: UFPel, 1996. cap.7, p.277304.

NALIM, D.M. Biologia, nutrição quantitativa e controle de qualidade de populações de Spodoptera frugiperda (J.E. Smith, 1797) (Lepidoptera: Noctuidae) em duas dietas artificiais. 1991. 150p. Tese (Doutorado) - Escola Superior de Agricultura Luiz de Queiroz, Piracicaba.

PARRA, J.R.P. Consumo e utilização de alimento por insetos. In: PANIZZI, A.R.; PARRA, J.R.P. (Ed.). Ecologia nutricional de insetos e suas implicações no manejo de pragas. São Paulo: Manole, 1991, p.9-65.

PARRA, J.R.P. Técnicas de criação de insetos para programas de controle biológico. Piracicaba: Esalq/USP, 2001. 134p.

PASHLEY, D.P. Host-associated genetic differentiation in fall armyworm (Lepidoptera: Noctuidae): a sibling species complex? Annals of the Entomological Society of America, v.79, p.898904, 1986.

PASHLEY, D.P.; MARTIN, J.A. Reproductive incompatibility between host strains of the fall armyworm (Lepidoptera: Noctuidae). Annals of the Entomological Society of America, v.80, p.731733, 1987.

PASHLEY, D.P.; QUISENBERRY, S.S.; JAMJANYA, T. Impact of fall armyworm (Lepidoptera: Noctuidae) host strains on the evaluation of bermuda grass resistance. Journal of Economic Entomology, v.80, p.1127-1130, 1987a.

PASHLEY, D.P.; SPARKS, T.C.; QUISENBERRY, S.S.; JAMJANYA, T.; DOWD, P.F. Two fall armyworm strains feed on corn, rice and bermuda grass. Louisiana Agriculture, v.30, p.8-9, 1987b.

SILVEIRA NETO, S.; NAKANO, O.; BARBIN, D.; VILLA NOVA, N.A. Manual de ecologia dos insetos. São Paulo: Ceres, 1976. 419p.

SINGH, P. A general purpose laboratory diet mixture for rearing insects. Insect Science, v.4, p.357-362, 1983.

SOUZA, A.M. de L. Adequação de recipientes, para criação em dietas artificiais, de pragas com comportamentos variáveis. 1999. 95p. Dissertação (Mestrado) - Escola Superior de Agricultura "Luiz de Queiroz”, Piracicaba.

WHITFORD, F.; QUISENBERRY, S.S.; RILEY, T.J.; LEE, J.W. Oviposition preference, mating compatibility, and development of two fall armyworm strains. Florida Entomologist, v.71, p.234243, 1988.

$\overline{\text { Recebido em } 12 \text { de fevereiro de } 2004 \text { e aprovado em } 24 \text { de novembro de } 2004}$ 